\title{
Manipulating crystallization dynamics through chelating molecules for bright perovskite emitters
}

\author{
Yatao Zou, ${ }^{1,2,12}$, Pengpeng Teng ${ }^{1,3,12}$, Weidong Xu (1) ${ }^{1 凶}$, Guanhaojie Zheng ${ }^{1}$, Weihua Lin ${ }^{4}$, Jun Yin (10) ${ }^{5}$, \\ Libor Kobera (1) ${ }^{6}$, Sabina Abbrent ${ }^{6}$, Xiangchun $\mathrm{Li}^{7}$, Julian A. Steele ${ }^{8}$, Eduardo Solano (i) ${ }^{9}$, \\ Maarten B. J. Roeffaers ${ }^{8}$, Jun Li (iD ${ }^{4}$, Lei Cai ${ }^{2}$, Chaoyang Kuang (iD) ${ }^{1}$, Ivan G. Scheblykin (i) ${ }^{4}$, Jiri Brus ${ }^{6}$, \\ Kaibo Zheng 4,10, Ying Yang 3 , Omar F. Mohammed (D) 5, Osman M. Bakr (D) 5, Tönu Pullerits (1D 4, Sai Bai (D) 1,11, \\ Baoquan Sun (i) ${ }^{2 \otimes} \&$ Feng Gao (i) ${ }^{1 凶}$
}

Molecular additives are widely utilized to minimize non-radiative recombination in metal halide perovskite emitters due to their passivation effects from chemical bonds with ionic defects. However, a general and puzzling observation that can hardly be rationalized by passivation alone is that most of the molecular additives enabling high-efficiency perovskite light-emitting diodes (PeLEDs) are chelating (multidentate) molecules, while their respective monodentate counterparts receive limited attention. Here, we reveal the largely ignored yet critical role of the chelate effect on governing crystallization dynamics of perovskite emitters and mitigating trap-mediated non-radiative losses. Specifically, we discover that the chelate effect enhances lead-additive coordination affinity, enabling the formation of thermodynamically stable intermediate phases and inhibiting halide coordination-driven perovskite nucleation. The retarded perovskite nucleation and crystal growth are key to high crystal quality and thus efficient electroluminescence. Our work elucidates the full effects of molecular additives on PeLEDs by uncovering the chelate effect as an important feature within perovskite crystallization. As such, we open new prospects for the rationalized screening of highly effective molecular additives.

\footnotetext{
${ }^{1}$ Department of Physics, Chemistry and Biology (IFM), Linköping University, Linköping, Sweden. ${ }^{2}$ Jiangsu Key Laboratory for Carbon-Based Functional Materials and Devices, Institute of Functional Nano and Soft Materials (FUNSOM), Joint International Research Laboratory of Carbon-Based Functional Materials and Devices, Soochow University, Suzhou, Jiangsu, People's Republic of China. ${ }^{3}$ State Key Laboratory of Mechanics and Control of Mechanical Structures, Nanjing University of Aeronautics and Astronautics, Nanjing, China. ${ }^{4}$ Chemical Physics and NanoLund, Lund University, Lund, Sweden. ${ }^{5}$ Division of Physical Science and Engineering, King Abdullah University of Science and Technology, Thuwal, Kingdom of Saudi Arabia. ${ }^{6}$ Institute of Macromolecular Chemistry of the Czech Academy of Sciences, Prague 6, Czech Republic. ${ }^{7}$ Key Laboratory for Organic Electronics and Information Displays, Institute of Advanced Materials (IAM), Jiangsu National Synergetic Innovation Center for Advanced Materials (SICAM), Nanjing University of Posts \&

Telecommunications, Nanjing, China. ${ }^{8}$ CMACS, Department of Microbial and Molecular Systems, KU Leuven, Leuven, Belgium. ${ }^{9}$ NCD-SWEET beamline, ALBA Synchrotron Light Source, Barcelona, Spain. ${ }^{10}$ Department of Chemistry, Technical University of Denmark, Kongens Lyngby, Denmark. ${ }^{11}$ State Key Lab of Silicon Materials, Zhejiang University, Hangzhou, P. R. China. ${ }^{12}$ These authors contributed equally: Yatao Zou, Pengpeng Teng. ${ }^{凶} \mathrm{email}$ : weidong.xu@liu.se; bqsun@suda.edu.cn; feng.gao@liu.se
} 
ntroducing molecular additives into perovskite precursors has become one of the most effective and prevailing strategies to improve the performance of metal halide perovskite lightemitting diodes (PeLEDs), and recently has boosted the external quantum efficiency (EQE) to high values above $\sim 20 \%{ }^{1-7}$. The performance enhancement results from suppressed nonradiative recombination and/or improved light-out coupling efficiency due to morphological variation ${ }^{6,8,9}$. The former is generally believed to be associated with defect passivation-surface dangling bonds in perovskites are said to be healed by additional coordination or ionic bonding with the additives ${ }^{1,10,11}$, leading to the elimination of trap states. Accordingly, a wide range of passivating molecular additives has been investigated for PeLEDs, especially those containing Lewis base moieties such as amino- and carboxylfunctionalized molecules ${ }^{8,12-15}$. In addition, the critical factors underlying passivation effectiveness have been well identified, including hardness/softness of functional moieties ${ }^{16}$, steric hindrance determined by molecular configurations ${ }^{17,18}$, as well as hydrogen bonding between passivating functional groups and organic cations ${ }^{7,19}$.

Despite these advances in understanding molecular passivation, a widely observed phenomenon yet to be rationalized by passivation alone is that almost all the effective additives for perovskite emitters are chelating molecules that contain more than one electron-rich functional moiety ${ }^{6,7,20}$. Conversely, monofunctionalized counterparts have received far less attention despite exhibiting similar molecular configurations and identical passivating groups. This contrast implies additional factors are at play (beyond the influence of passivation) that critically affect radiative recombination in the resulting halide perovskites.

Inspirations can be drawn from recent work on perovskite photovoltaics and point toward the possibility that molecular additives can modify crystallization dynamics ${ }^{21,22}$, resulting in discrepancies in defect densities and hence different radiative recombination processes. For instance, modulating crystallization dynamics with Lewis basic solvents, such as dimethyl sulfoxide (DMSO) and 1-methyl-2-pyrrolidinone (NMP), have been intensively investigated ${ }^{21-23}$. However, the chemical composition of perovskite emitters is remarkably different from that of perovskite photovoltaics due to the required enhancement of quantum and/or dielectric confinement ${ }^{4,24-26}$. Consequently, the crystallization control strategies developed in perovskite photovoltaics cannot be expected to be straightforwardly translated to PeLEDs. A thorough understanding of how the molecular additives influence perovskite crystallization in PeLEDs is thus required to better rationalize where the optoelectronic improvements originate.

Here, we reveal that the effects of prevailing molecular additives on manipulating the crystallization process of perovskite emitters are largely underestimated. By comparing two highly effective chelating molecules with their respective monofunctionalized counterparts, we find that chelation enhanced lead-additive binding affinity enables the formation of thermodynamically stable intermediates, leading to effective inhibition of perovskite nucleation and retarded crystal growth. The retarded crystallization process results in highly emissive perovskite thin films and high-efficiency near-infrared (NIR) PeLEDs with EQEs over $20 \%$. We further generalize our conclusions by investigating a wide range of additives with different coordination numbers and functional moieties. Our results fill the knowledge gap between chelating molecular additives and bright perovskite emitters, through establishing the correlation between leadadditive coordination affinity and perovskite crystallization dynamics. As such, we provide a universal guideline for tuning the crystallization dynamics for high-performance PeLEDs by leveraging the chelate effect.

\section{Results}

PeLED device performance and film characterization. We compare the effects of two popular chelating additives (CAs) with their respective mono-functionalized additives (MFAs) on formamidinium lead tri-iodide $\left(\mathrm{FAPbI}_{3}\right)$ emitters. The CAs we use are 5-aminovaleric acid (5AVA) and 3,6,9,12-tetraoxatetradecane-1,14-diamine $\left(\mathrm{NH}_{2}-\mathrm{PEG}_{4}-\mathrm{NH}_{2}\right)$, which consist of commonly used amino- and/or carboxyl- groups; their respective mono-functionalized counterparts are $n$-butylamine (BA) and propionic acid (PA) for the former, and 2-(2-methoxyethoxy)ethanamine $\left(\mathrm{m}-\mathrm{PEG}_{2}-\mathrm{NH}_{2}\right.$ ) for the latter (Fig. 1a). Here, $\mathrm{m}-\mathrm{PEG}_{2}-\mathrm{NH}_{2}$ is regarded as an MFA despite the electrondonating nature of the $\mathrm{O}$ atoms. This is based on the fact that the effects of oxygen atoms on crystallization are not as prominent as amino groups, as verified by comparing a counterpart with a longer polyethylene glycol (PEG) chain (2,5,8,11-tetraoxatridecan-13-amine, $\mathrm{m}-\mathrm{PEG}_{4}-\mathrm{NH}_{2}$ ) (Supplementary Fig. 1 and Supplementary Note 1). All the additives were introduced into perovskite precursors at a mole ratio of $\mathrm{PbI}_{2}$ :formamidinium iodide (FAI):additive $=1: 2: x \quad(x=0-0.8)$. The use of excess organic components aims to passivate the vacancy defects, as widely demonstrated in PeLEDs ${ }^{27,28}$. As shown in the X-ray diffraction (XRD) patterns (Supplementary Fig. 2), the addition of additives does not change the three-dimensional structure of $\mathrm{FAPbI}_{3}$ at the optimal feed ratio for device fabrication. Besides, all the perovskite films show nano-island morphological features, although the addition of CA leads to smaller crystal sizes and denser surface coverage compared to the MFA-based counterparts (Supplementary Fig. 3). Our further investigations indicate that the morphological differences between MFA- and CA-films cannot be straightforwardly linked to the discrepancies in the device performance (Supplementary Fig. 4 and Supplementary Note 2).

We fabricate PeLEDs with additives and that without (named as the "control") by subsequential deposition of zinc oxide ( $\mathrm{ZnO} ; 10$ $\mathrm{nm}) /$ polyethyleneimine ethoxylated (PEIE)/FAPbI $3 /$ poly $(9,9-$ dioctylfluorene-co-N-(4-(3-methylpropyl)) diphenylamine (TFB)/ $\mathrm{MoO}_{3}(7 \mathrm{~nm}) / \mathrm{Au}(80 \mathrm{~nm})$ on indium tin oxide (ITO) substrates (see Fig. $1 \mathrm{~b}$ and a cross-sectional image in Supplementary Fig. 5a). Devices based on the combined use of BA and PA at the mole ratio of 1:1 (named as the "BA-PA") are also studied for comparison. The energy levels of the perovskite films are determined by ultraviolet photoelectron spectroscopy (UPS) spectra and a flat-band energy level diagram of devices (with control, $\mathrm{m}-\mathrm{PEG}_{2}-\mathrm{NH}_{2}$ and $\mathrm{NH}_{2}-\mathrm{PEG}_{4}-\mathrm{NH}_{2}$ devices as the examples) are shown in Supplementary Fig. 5b-d and further discussed in Supplementary Note 3.

As the additives reduce the charge carrier injection to some extent due to their insulating nature (Supplementary Fig. 6), we carefully control their loading contents (see the details in Supplementary Table 1) to maximize the peak EQEs and simultaneously ensure a radiance as large as possible. We show a summary of average EQE values and detailed performance parameters with the optimized feed ratio in Fig. 1c and Supplementary Table 2, respectively, from which we find that $\mathrm{CA}$ addition results in considerable enhancement in terms of both radiance and EQE values compared to control and the respective MFA counterparts. Specifically, control devices show an average peak EQE of $5.3 \pm 0.7 \%$; $5 \mathrm{AVA}$-devices give an average peak EQE of $15.5 \pm 0.8 \%$ compared to $7.1 \pm 0.7 \%, 6.5 \pm 0.6 \%$, and $7.6 \pm 0.5 \%$ of $\mathrm{BA}, \mathrm{PA}$, and $\mathrm{BA}-\mathrm{PA}$ mixture, respectively; $\mathrm{NH}_{2}-\mathrm{PEG}_{4}-\mathrm{NH}_{2}$ devices have an average peak EQE of $19.2 \pm$ $0.6 \%$ compared to $11.0 \pm 0.5 \%$ of $\mathrm{m}-\mathrm{PEG}_{2}-\mathrm{NH}_{2}$. The $\mathrm{NH}_{2}-\mathrm{PEG}_{4}-\mathrm{NH}_{2}-$ and $5 \mathrm{AVA}$-devices exhibit average radiance values of $360 \pm 27 \mathrm{~W} \mathrm{sr}^{-1} \mathrm{~m}^{-2}$ and $173 \pm 20 \mathrm{~W} \mathrm{sr}^{-1} \mathrm{~m}^{-2}$, which contrast sharply with the respective MFA devices (Supplementary 


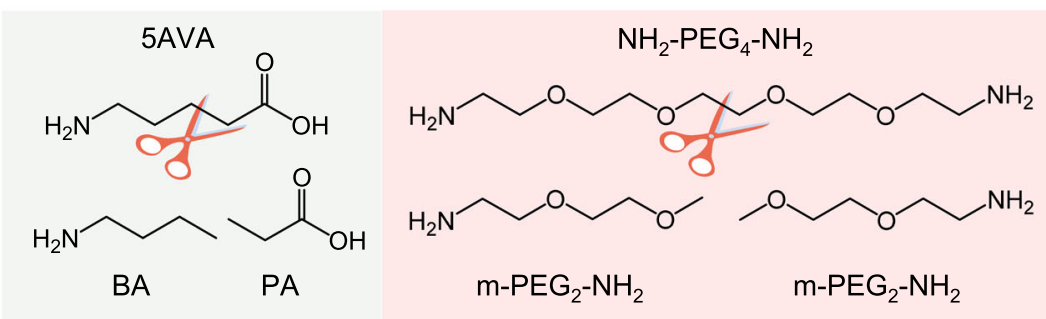

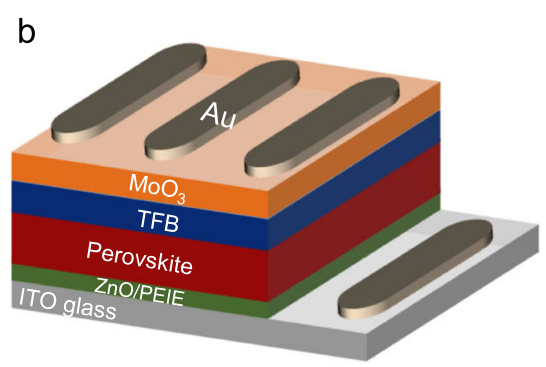
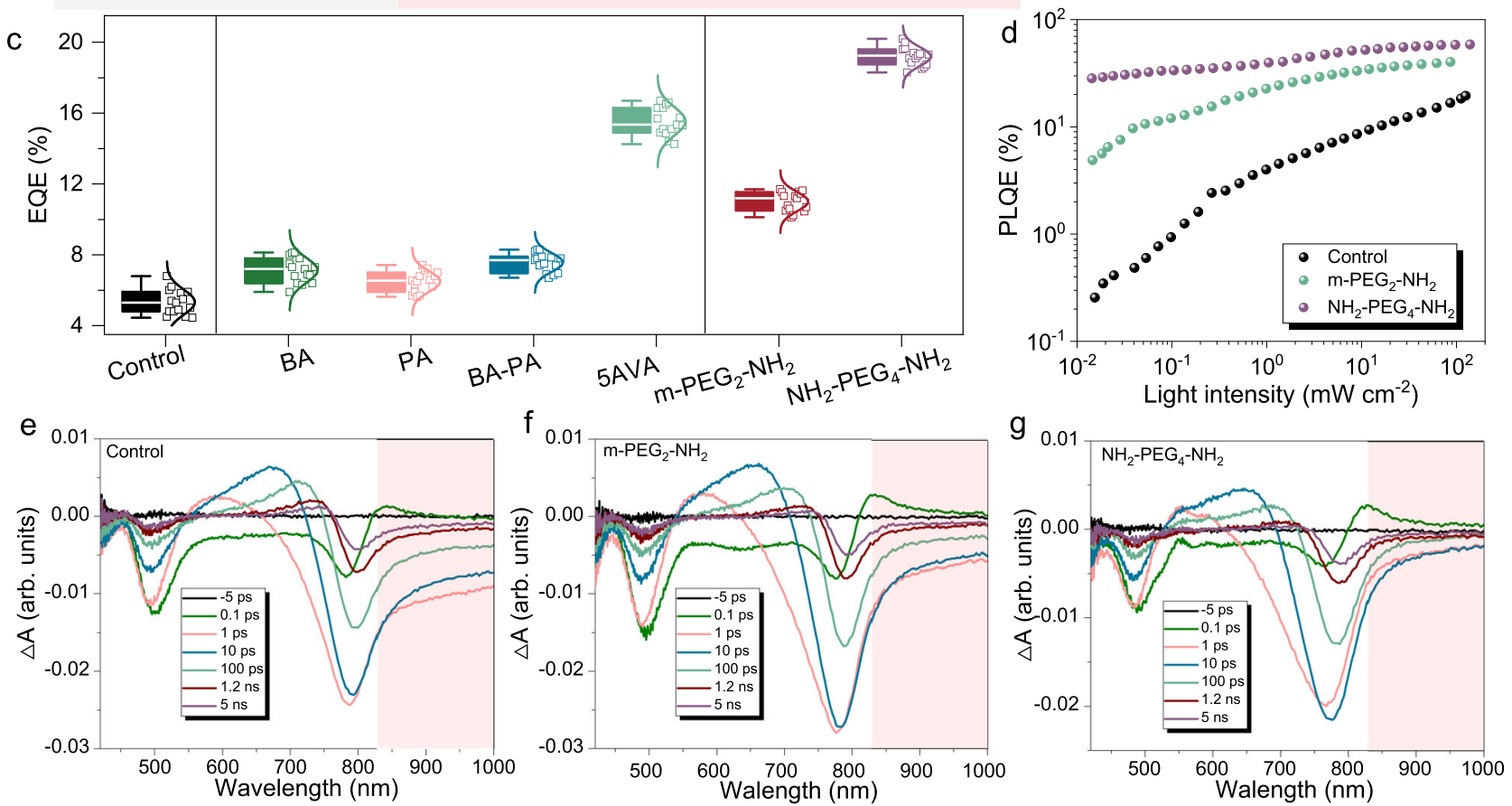

Fig. 1 PeLED device architecture, performance, and perovskite film characteristics. a Molecular structures of the candidate chelating molecules (5AVA and $\mathrm{NH}_{2}-\mathrm{PEG}_{4}-\mathrm{NH}_{2}$ ), and their respective mono-functionalized counterparts (BA, $\mathrm{PA}$, and $\mathrm{m}-\mathrm{PEG} \mathrm{NH}_{2}$ ). b $\mathrm{A}$ schematic of PeLED architecture. c EQE statistical distribution of 20 devices fabricated with different additives. $\mathbf{d}-\mathbf{g}$ Photophysical studies for control, $\mathrm{m}_{-}-\mathrm{PEG}_{2}-\mathrm{NH}_{2}$, and $\mathrm{NH}_{2}-\mathrm{PEG}_{4}-\mathrm{NH}_{2} \mathrm{Perovskite}$ thin films: fluence-dependent PLQE (d); TA spectra of control perovskite films (e), and the films with $\mathrm{m}-\mathrm{PEG}_{2}-\mathrm{NH}_{2}(\mathbf{f})$ and $\mathrm{NH}_{2}-\mathrm{PEG}{ }_{4}-\mathrm{NH}_{2}(\mathbf{g})$. The pink squares in (e-g) are to highlight the band tail absorption.

Table 2). In addition, the champion $\mathrm{NH}_{2}-\mathrm{PEG}_{4}-\mathrm{NH}_{2^{-}}$and $5 \mathrm{AVA}$-devices reach high EQE values of $20.2 \%$ and $17.2 \%$, respectively (see device characteristics in Supplementary Figs. 7 and 8). Intriguingly, BA-PA mixed precursors and double amounts of $\mathrm{m}-\mathrm{PEG}_{2}-\mathrm{NH}_{2}$ do not give rise to comparable performance as the respective CA counterpart, suggesting the presence of additional underlying factors determining the device performance in addition to the amounts of Lewis base moieties. Moreover, CA devices show a prolonged operational lifetime with respect to the MFA counterparts measured under a constant current density of $20 \mathrm{~mA} \mathrm{~cm}^{-2}$ (Supplementary Fig. 9).

Given that $\mathrm{m}-\mathrm{PEG}_{2}-\mathrm{NH}_{2}$ shares identical moieties with $\mathrm{NH}_{2}-\mathrm{PEG}_{4}-\mathrm{NH}_{2}$, we choose them as the main candidates for the rest of our investigations. We explore the photophysical properties of the perovskite films to further evaluate the radiative recombination and provide a rationale for the superior performance of $\mathrm{CA}$ devices. As shown in Fig. 1d, the $\mathrm{NH}_{2}-\mathrm{PEG}_{4}-\mathrm{NH}_{2}$ films display a much less pronounced dependence of photoluminescence quantum efficiencies (PLQEs) on excitation fluences with respect to its MFA counterparts and control films, giving the highest peak PLQE value up to $50 \%$. More efficient radiative recombination in $\mathrm{NH}_{2}-\mathrm{PEG}_{4}-\mathrm{NH}_{2}$ films is also evident in PL mapping measurements ${ }^{29,30}$, from which we observe very homogeneous emission in space for the $\mathrm{NH}_{2}-\mathrm{PEG}_{4}-\mathrm{NH}_{2}$ film with time but distinct PL blinking in the control and $\mathrm{m}-\mathrm{PEG}_{2}-\mathrm{NH}_{2}$ cases (Supplementary Fig. 10 and Supplementary Movies 1-3).

To further understand the discrepancy in the PL properties, we proceed to investigate the electronic states of the perovskite films using transient absorption (TA) measurements (Fig. 1e-g). In all the cases, we observe two photobleaching $(\mathrm{PB})$ bands situated near $780 \mathrm{~nm}$ (PB1) and $490 \mathrm{~nm}$ (PB2), suggesting no significant alteration in their photophysical behavior with additives addition. The appearance of $\mathrm{PB} 2$ has been previously observed in bulk $\mathrm{CH}_{3} \mathrm{NH}_{3} \mathrm{PbI}_{3}$ perovskites, but the assignment remains unclear $^{31,32}$. One previous report proposes that PB1 and PB2 arise from the transitions between two valence bands (VBs) to a common conduction band minimum $(\mathrm{CBM})^{31}$. Notably, the control film shows a distinct band $(>800 \mathrm{~nm})$ in different delayed time scales, suggesting a relatively large number of sub-bandgap trap states ${ }^{33}$. The band tail absorption is reduced to some extent in $\mathrm{m}-\mathrm{PEG}_{2}-\mathrm{NH}_{2}$ films but remarkably weakened in $\mathrm{NH}_{2}-\mathrm{PEG}_{4}-\mathrm{NH}_{2}$ incorporated films. Following the decreases in the band tail absorption, the PB1 is slightly blue-shifted compared to control and $\mathrm{m}-\mathrm{PEG}_{2}-\mathrm{NH}_{2}$ cases, which can be assigned to the decoupling between $\mathrm{PB} 1$ and the less pronounced sub-bandgap trap states. Combining the results of PLQEs, PL mapping, and TA measurements, we conclude that trap-assisted nonradiative recombination is largely suppressed in $\mathrm{NH}_{2}-\mathrm{PEG}_{4}-\mathrm{NH}_{2}$ films, resulting in superior device performance. 

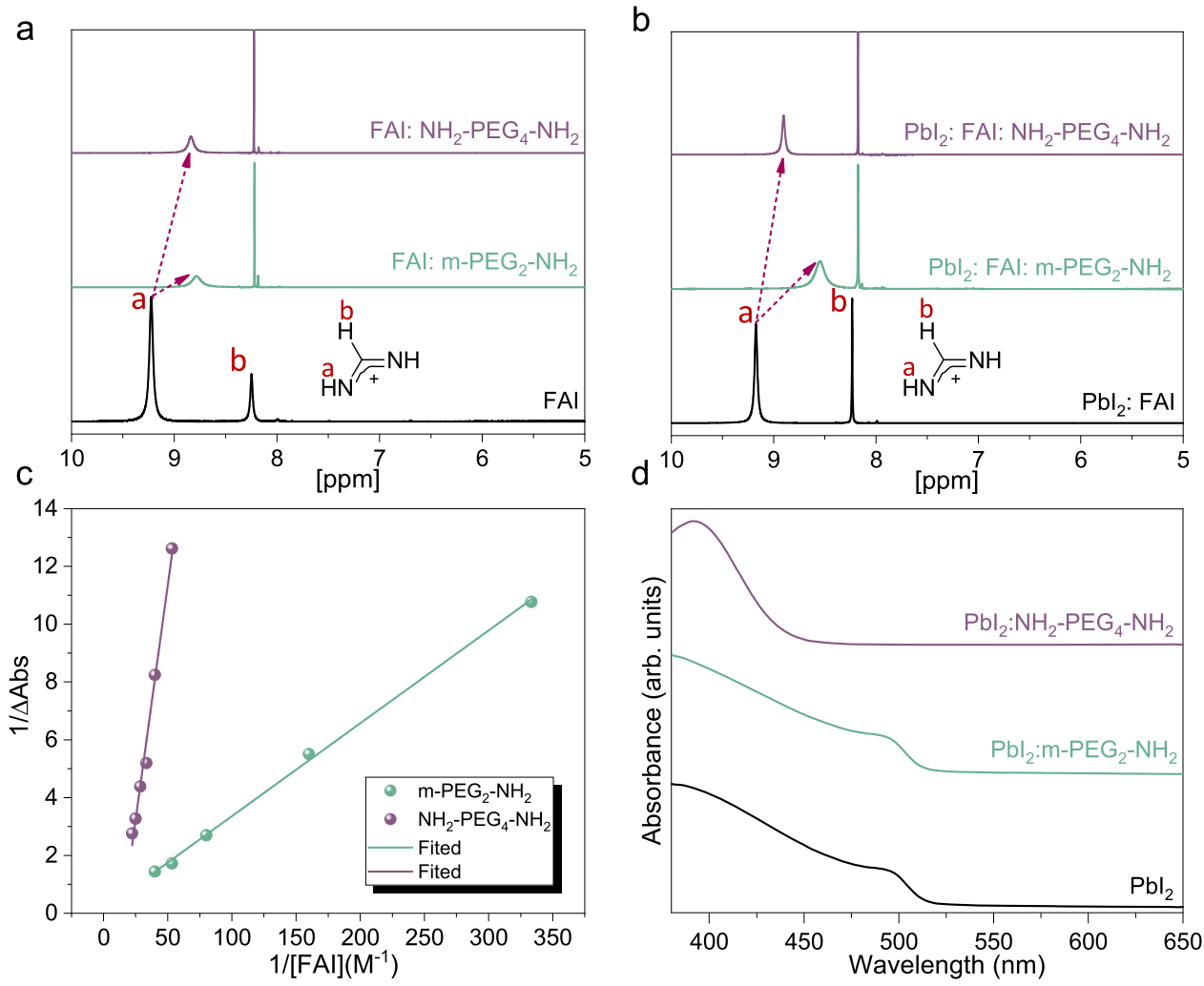

Fig. 2 Interactions between the additives and perovskite precursors. $\mathbf{a}, \mathbf{b}{ }^{1} \mathrm{H} N M R$ of $F A I, F A I: m-P E G_{2}-N_{2}(1: 0.2)$ and $F A l: N H_{2}-P E G_{4}-N H_{2}(1: 0.1)(\mathbf{a})$ and $\mathrm{Pbl}_{2}: \mathrm{FAl}$ (1:2), $\mathrm{Pbl}_{2}: \mathrm{FAl}: \mathrm{m}-\mathrm{PEG}_{2}-\mathrm{NH}_{2}(1: 2: 0.4)$ and $\mathrm{Pbl}_{2}: \mathrm{FAl}: \mathrm{NH}_{2}-\mathrm{PEG}_{4}-\mathrm{NH}_{2}(1: 2: 0.2)$ (b). Peaks a and $\mathrm{b}$ are signals of $\mathrm{H}(\mathbf{a})$ and $\mathrm{H}(\mathbf{b})$ as labeled in the chemical structure of $\mathrm{FA}^{+}$in the inset. c BH plots extracted from the traces of $\mathrm{Pbl}_{3}{ }^{-}$absorption ( $370 \mathrm{~nm}$ ) with increasing $\mathrm{FAl}$ content in $\mathrm{Pbl}_{2}$ : $\mathrm{NH}_{2}-\mathrm{PEG}_{4}-\mathrm{NH}_{2}$ and $\mathrm{Pbl}_{2}: \mathrm{m}-\mathrm{PEG}_{2}-\mathrm{NH}_{2}$ parental solutions (Supplementary Fig. 16). d Ultraviolet-visible (UV-vis) absorption of neat Pbl $\mathrm{Pb}_{2}$ film, $\mathrm{Pbl}$ : $\mathrm{m}$ $\mathrm{PEG}_{2}-\mathrm{NH}_{2}$ (1:0.4), and $\mathrm{Pbl}_{2}: \mathrm{NH}_{2}-\mathrm{PEG}_{4}-\mathrm{NH}_{2}$ (1:0.2) films. All the stoichiometric ratios here are mole ratios.

Understanding the effects of additives. The reduced trapassisted nonradiative recombination could be attributed to efficient defect passivation, improved crystal quality, or a collective effect of them. By Fourier transform-infrared spectroscopy (FTIR) measurements, we demonstrate that both $\mathrm{m}-\mathrm{PEG}_{2}-\mathrm{NH}_{2}$ and $\mathrm{NH}_{2}-\mathrm{PEG}_{4}-\mathrm{NH}_{2}$ are retained in the perovskite films, as confirmed by the appearance of stretching vibration of ether bonds (C-O-C) at around $1110 \mathrm{~cm}^{-1}$ (Supplementary Fig. 11). The presence of additives provides opportunities for them to interact with defects chemically, contributing to the mitigated nonradiative recombination. It is further confirmed by the density of functional theory (DFT) calculations (Supplementary Figs. 12 and 13). Notably, our theoretical calculations suggest little differences in passivation effectiveness between these two additives, as further discussed in Supplementary Fig. 13 and Supplementary Note 4. This is also consistent with the fact that the passivation effects are usually determined by the type of functional groups and their chemical environment, both of which are identical in $\mathrm{m}$ $\mathrm{PEG}_{2}-\mathrm{NH}_{2}$ and $\mathrm{NH}_{2}-\mathrm{PEG}_{4}-\mathrm{NH}_{2}$, as confirmed by calculation of the electronic property of the molecules (Supplementary Fig. 14). These results exclude the possibility that the significant difference in radiative recombination and device performance are the results of different passivation effectiveness between these two molecules.

With little difference in passivation effectiveness expected to occur between the use of CA and MFA additives, we focus our investigations on the effects of different molecules on the crystallization process. The first question that arises is whether these additives have different interaction strengths with the perovskite precursors; the interaction strength determines the intermediate phases and thus plays the key role in governing the crystallization process $21,34,35$. Amines can form hydrogen bonds with charged ammonium cations $\left(\mathrm{FA}^{+}\right)^{7}$, which are characterized by ${ }^{1} \mathrm{H}$ NMR. The number of Lewis base moieties is constant in all the samples, that is, at a mole ratio of 1:0.2 for FAI: m-PEG ${ }_{2}-\mathrm{NH}_{2}$ mixtures and 1:0.1 for FAI: $\mathrm{NH}_{2}-\mathrm{PEG}_{4}-\mathrm{NH}_{2}$ ones. The resonance signal of protonated ammonium peaks at $9.3 \mathrm{ppm}$ in the pure FAI deuterated-DMF solution (Fig. 2a). In both cases with additive addition, the resonance signals of ammonium shift to $8.8 \mathrm{ppm}$, implying similar interaction strengths between both additives and $\mathrm{FA}^{+}$. These results are in line with the DFT calculations that the chemical environments of the amino groups and oxygen atoms in $\mathrm{m}-\mathrm{PEG}_{2}-\mathrm{NH}_{2}$ and $\mathrm{NH}_{2}-\mathrm{PEG}_{4}-\mathrm{NH}_{2}$ are very close. In contrast, for the samples containing $\mathrm{PbI}_{2}$, the resonance of ammonium becomes less susceptible to $\mathrm{NH}_{2}-\mathrm{PEG}_{4}-\mathrm{NH}_{2}$ addition compared to $\mathrm{m}-\mathrm{PEG}_{2}-\mathrm{NH}_{2}$, as adding the latter results in a much larger shift toward high field (Fig. 2b). These results imply that $\mathrm{NH}_{2}-\mathrm{PEG}_{4}-\mathrm{NH}_{2}$ has a stronger tendency than $\mathrm{m}-\mathrm{PEG}_{2}-\mathrm{NH}_{2}$ to work with $\mathrm{Pb}^{2+}$ and thus leads to a weaker interaction with $\mathrm{FA}^{+}$in the $\mathrm{Pb}^{2+}$-contained precursor solutions.

In this regard, we proceed to directly investigate the interaction between the additives and $\mathrm{Pb}^{2+}$. Adding $\mathrm{NH}_{2}-\mathrm{PEG}_{4}-\mathrm{NH}_{2}$ into $\mathrm{PbI}_{2}$ solution leads to the formation of white precipitation (Supplementary Fig. 15). This observation is consistent with the fact that amines are well-known building blocks for constructing metal amide complexes. Moreover, the yielded products will gradually diminish with FAI addition, indicating a competitive ligand exchange between the electron-rich moieties of additives and iodide anions from FAI.

We next quantified the interaction strength between Lewis base ligands and $\mathrm{Pb}^{2+}$ on the basis that iodide ions compete with 
Lewis base moieties to coordinate with $\mathrm{Pb}^{2+}$ ions ${ }^{36}$. We perform Benesi-Hildebrand $(\mathrm{BH})$ analyses and determine the formation constant $\left(K_{\mathrm{f}}\right)$ of iodoplumbate complex $\mathrm{PbI}_{3}{ }^{-}$in the solution of interes $\mathrm{t}^{37}$, by monitoring the Ultraviolet-visible (UV-vis) absorption evolution with increasing FAI content in the $\mathrm{PbI}_{2}$ :additive parental solutions. We double the amounts of $\mathrm{m}-\mathrm{PEG}_{2}-\mathrm{NH}_{2}$ with regards to $\mathrm{NH}_{2}-\mathrm{PEG}_{4}-\mathrm{NH}_{2}$ to keep Lewis base moieties constant in the solutions. By following the absorption spectra evolution with increasing FAI content, we observe gradually enhanced absorption intensity of $\mathrm{PbI}_{3}{ }^{-}$in both cases (Supplementary Fig. 16). Notably, $\mathrm{PbI}_{2}: \mathrm{NH}_{2}-\mathrm{PEG}_{4}-\mathrm{NH}_{2}$ parental solution requires much higher amounts of FAI to achieve comparable absorbance to $\mathrm{m}-\mathrm{PEG}_{2}-\mathrm{NH}_{2}$ ones. Figure $2 \mathrm{c}$ shows the $\mathrm{BH}$ plots, from which our calculations yield a larger $K_{\mathrm{f}}$ value (1/slope) of $31.1 \mathrm{M}^{-1}$ for $\mathrm{m}-\mathrm{PEG}_{2}-\mathrm{NH}_{2}$ solution compared to $3.1 \mathrm{M}^{-1}$ for that with $\mathrm{NH}_{2}-\mathrm{PEG}_{4}-\mathrm{NH}_{2}$. These results confirm that the iodoplumbate complex is more difficult to form with the presence of $\mathrm{NH}_{2}-\mathrm{PEG}_{4}-\mathrm{NH}_{2}$, indicating a higher affinity between $\mathrm{NH}_{2}-\mathrm{PEG}_{4}-\mathrm{NH}_{2}$ and $\mathrm{Pb}^{2+}$. We attribute this to the chelate effect that the chelating ligands (e.g. $\mathrm{NH}_{2}-\mathrm{PEG}_{4}-\mathrm{NH}_{2}$ ) have a stronger affinity to the metal ions than a collection of their nonchelating or weak chelating counterparts (e.g. $\mathrm{m}-\mathrm{PEG}_{2}-\mathrm{NH}_{2}$ ) despite identical functional groups, leading to kinetically more stable lead-additive coordination in the solutions.

Another important effect of the $\mathrm{Pb}^{2+}-\mathrm{CA}$ coordination with chelation enhanced affinity is the formation of thermodynamically stable lead complexes in the solid states. We show the UV-vis absorption spectra of $\mathrm{PbI}_{2}$ : additive and neat $\mathrm{PbI}_{2}$ films in Fig. 2d and their XRD patterns in Supplementary Fig. 17. All these films were annealed at $100{ }^{\circ} \mathrm{C}$ for $10 \mathrm{~min}$ before measurements. Compared to the neat $\mathrm{PbI}_{2}$ film, the $\mathrm{PbI}_{2}$ : $\mathrm{NH}_{2}-\mathrm{PEG}_{4}-\mathrm{NH}_{2}$ film turns to colorless (Supplementary Fig. 18), where the absorption onset shifts to a shorter wavelength of around $\sim 450 \mathrm{~nm}$ (Fig. 2d). It also displays an amorphous feature with no discernible diffraction peaks, in sharp contrast to the $\mathrm{PbI}_{2}$ neat film which shows a typical crystalline (110) diffraction peak at $2 \theta=12.6^{\circ}$ (Supplementary Fig. 17). These results suggest that the addition of $\mathrm{NH}_{2}-\mathrm{PEG}_{4}-\mathrm{NH}_{2}$ reconstructs $\mathrm{Pb}^{2+}$ coordination to form complexes that are stable at high temperatures for perovskite crystallization. In contrast, the $\mathrm{PbI}_{2}: \mathrm{m}-\mathrm{PEG}_{2}-\mathrm{NH}_{2}$ film shows no difference compared to the neat $\mathrm{PbI}_{2}$ film regarding both absorption and XRD patterns, indicating that $\mathrm{m}-\mathrm{PEG}_{2}-\mathrm{NH}_{2}$ only has labile coordination to lead cations and that the formed complexes are readily decomposed at high temperatures needed for annealing perovskite films. We observe similar results in 5AVA and its MFA counterparts (BA, PA, and BA-PA combination). In particular, 5AVA: $\mathrm{PbI}_{2}$ films show identical blue-shifted absorption spectra and amorphous features as the $\mathrm{NH}_{2}-\mathrm{PEG}_{4}-\mathrm{NH}_{2}$ cases, while $\mathrm{BA}, \mathrm{PA}$, and $\mathrm{BA}-\mathrm{PA}$ additions give no difference compared to neat $\mathrm{PbI}_{2}$ films (Supplementary Fig. 19).

The formation of kinetically and thermodynamically more stable complexes, both attributed to the strong affinity of CAs to $\mathrm{Pb}^{2+}$, collectively affect the crystallization process of perovskites. Our characterizations show that the additives which exhibit a stronger affinity to lead cations are more effective in suppressing perovskite nucleation. The XRD patterns for the control and $\mathrm{m}$ $\mathrm{PEG}_{2}-\mathrm{NH}_{2} \quad\left(\mathrm{PbI}_{2}: \mathrm{FAI}: \mathrm{m}-\mathrm{PEG}_{2}-\mathrm{NH}_{2}=1: 2: 0.2\right)$ precursor films before annealing display the diffraction peaks from $\alpha$-phase $\mathrm{FAPbI}_{3}$ (Supplementary Fig. 20a, b). We observe broad PL emission bands (Supplementary Fig. 21) peaking at shorter wavelengths compared to the typical 3D $\mathrm{FAPbI}_{3}$ in the control and $\mathrm{m}-\mathrm{PEG}_{2}-\mathrm{NH}_{2}$-based precursor films, as well as two distinct excitonic absorption features peaking at $\sim 450$ and $\sim 495 \mathrm{~nm}$ (Fig. 3a). Although the assignment of $\sim 450 \mathrm{~nm}$ excitonic absorption is not clear, the other results clearly suggest the formation of $\alpha-\mathrm{FAPbI}_{3}$ nanocrystals with size smaller than the Bohr radius. This can be directly observed by the transmittance
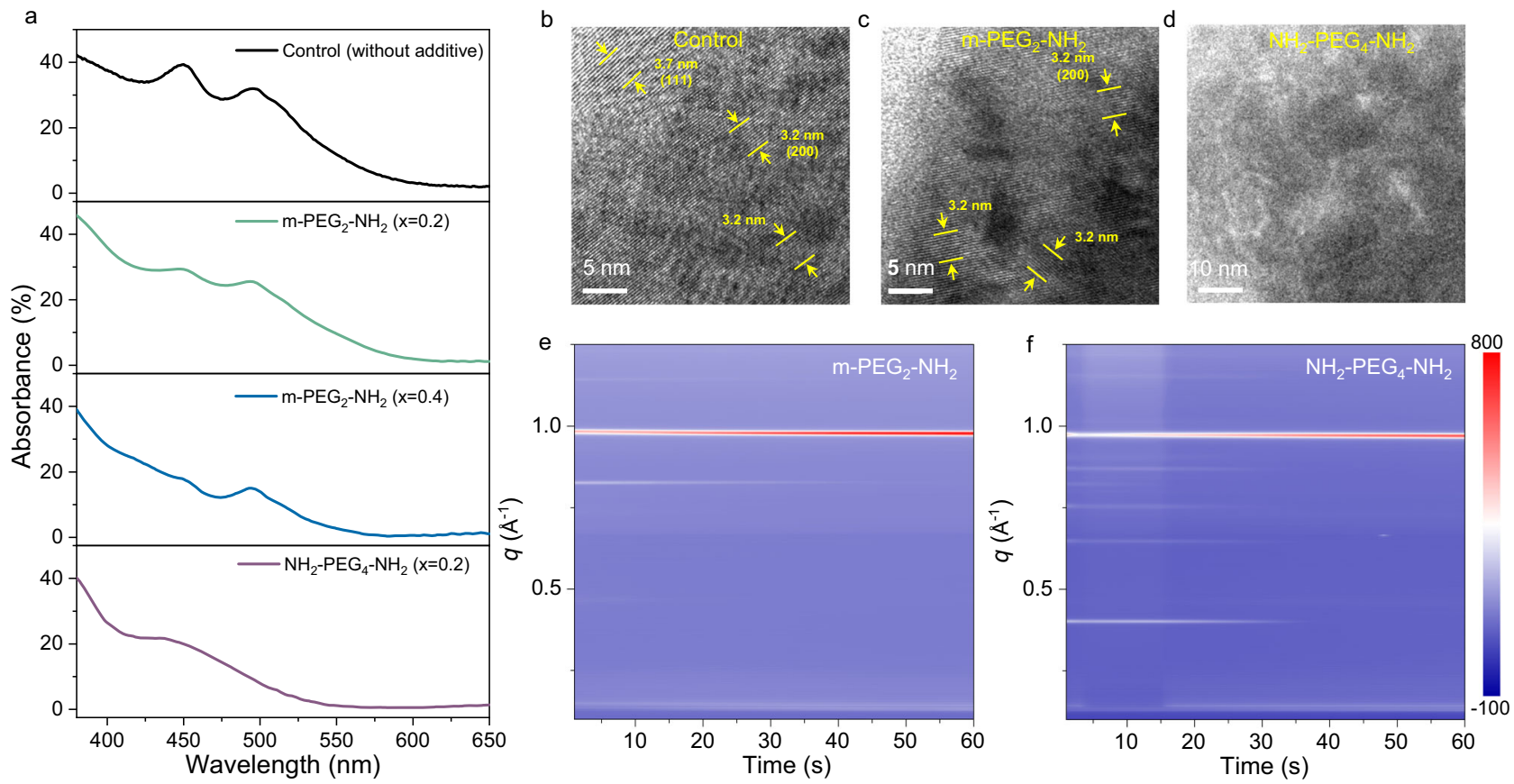

Fig. 3 Analysis of perovskite nucleation and crystallization dynamics. a Ultraviolet-visible (UV-vis) absorption spectra of the control, $\mathrm{Pbl} \mathrm{I}_{2}: \mathrm{FAl}: \mathrm{m}$ $\mathrm{PEG}_{2}-\mathrm{NH}_{2}$ (1:2:0.2 and 1:2:0.4) and $\mathrm{Pbl}_{2}: \mathrm{FAl}: \mathrm{NH}_{2}-\mathrm{PEG}_{4}-\mathrm{NH}_{2}$ (1:2:0.2) precursor films without annealing. b-d TEM images of the perovskite films before annealing. control (b); with $\mathrm{m}-\mathrm{PEG}_{2}-\mathrm{NH}_{2}(\mathbf{c})$; with $\mathrm{NH}_{2}-\mathrm{PEG}_{4}-\mathrm{NH}_{2}(\mathbf{d})$. e, $\mathbf{f}$ Diffraction intensity map versus $q$ with annealing time for perovskite films with a composition of $\mathrm{Pbl}_{2}: \mathrm{FAl}: \mathrm{m}-\mathrm{PEG}_{2}-\mathrm{NH}_{2}=1: 2: 0.4$ (e) and $\mathrm{Pbl}_{2}: \mathrm{FAl}: \mathrm{NH}_{2}-\mathrm{PEG}_{4}-\mathrm{NH}_{2}=1: 2: 0.2$ (f). The films were deposited on ITO/ZnO/PEIE substrates. All the stoichiometric ratios here are mole ratios. 
electron microscopy (TEM) results (Fig. 3b, c), where (100) and (200) planes of $\mathrm{FAPbI}_{3}$ crystals are discernable in control- and MFA-precursor samples. It is worthy to mention that the perovskite nanocrystals form during the film formation process rather than in the precursor solutions, as no "Tyndall effect" is visible in the precursor solutions (Supplementary Fig. 22). In comparison, no signature of crystalline perovskites can be found from XRD patterns (Supplementary Fig. 20c) and TEM images (Fig. 3d) in the optimal $\mathrm{NH}_{2}-\mathrm{PEG}_{4}-\mathrm{NH}_{2}$ precursor films without annealing $\left(\mathrm{PbI}_{2}: \mathrm{FAI}: \mathrm{NH}_{2}-\mathrm{PEG}_{4}-\mathrm{NH}_{2}=1: 2: 0.2\right)$. Consistently, we do not observe any discernible excitonic absorption features from UV-vis absorption spectra (Fig. 3a). Due to the absence of perovskites in the precursor films, no PL can be detected. All these results indicate that the perovskite nucleation is completely suppressed at room temperature with the addition of $\mathrm{NH}_{2}-\mathrm{PEG}_{4}-\mathrm{NH}_{2}$ by forming kinetically and thermodynamically stable intermediate phases. Notably, although these results indicate that chelating ligands are effective in suppressing perovskite nucleation, we notice that suppression of nucleation at room temperature does not necessarily indicate a good film quality, as simply increasing the amount of nonchelating additive can also suppress perovskite nucleation yet without benefiting the film quality (Supplementary Fig. 23).

We further investigate the in situ crystallization process with different additives by monitoring the grazing-incidence wideangle X-ray scattering (GIWAXS) pattern evolution during thermal annealing. Since the conversion from the intermediate phases to $\alpha-\mathrm{FAPbI}_{3}$ perovskites is very fast at a high temperature $\left(100^{\circ} \mathrm{C}\right)$ for device fabrication, we slow down the process by continuously increasing the temperature from 88 to $100^{\circ} \mathrm{C}$ in 60 s. We show the evolution of integrated diffraction intensities on annealing time in Fig. 3e, f. The m- $\mathrm{PEG}_{2}-\mathrm{NH}_{2}$ film (0.4 eq to lead content) initially shows two distinct scattering rings with $q$ value of $\sim 0.98$ and $\sim 0.83 \AA^{-1}$, which correspond to $\alpha-\mathrm{FAPbI}_{3}$ and nonperovskite $\delta-\mathrm{FAPb}_{3}$, respectively. The signatures of $\alpha-\mathrm{FAPb}_{3}$ and $\delta$ - $-\mathrm{FAPI}_{3}$ can be detected in the $\mathrm{NH}_{2}-\mathrm{PEG}_{4}-\mathrm{NH}_{2}$ sample at the initial stage as well, but with much weaker diffraction intensities, consistent with the XRD results that the perovskite nucleation can hardly occur at a low temperature (Supplementary Fig. 20c). In addition, five more weak diffraction signals at the $q$ values of $0.40,0.65,0.74,0.86$, and $0.90 \AA$ are visible, implying the coexistence of multiple crystalline intermediate phases during crystallization. Following the increase of annealing time, the diffraction signals from $\delta$-FAPbI 3 and intermediate phases gradually diminish until they eventually disappear, accompanied by the increases in $\alpha-\mathrm{FAPbI}_{3}$ diffraction intensity. Notably, a much longer time is required for the saturation of (110) plane diffraction peak in the $\mathrm{NH}_{2}-\mathrm{PEG}_{4}-\mathrm{NH}_{2}$ film compared to $\mathrm{m}$ $\mathrm{PEG}_{2}-\mathrm{NH}_{2}$. This is in line with the proposed competitive ligand exchange process that iodide anions need to replace Lewis base ligands to coordinate with lead cations, and thus higher energy is required for the formation of $\mathrm{PbI}_{6}{ }^{4-}$ octahedra and following perovskite nucleation ${ }^{38}$. In addition, we suggest that the ligand exchange process continually occurs during the crystallization process, leading to constrained crystal growth and hence small grain sizes (Supplementary Fig. 3). As such, we conclude that the chelating additives serve as more efficient crystallization inhibitors by leveraging their strong affinity to lead cations.

Investigations of other Lewis base additives. In order to complete the whole picture and generalize our findings, we investigate a wide range of Lewis base additives. We start with DMSO, the most intensively used Lewis base additive in perovskite photovoltaics, where a colorless DMSO-PbI 2 -FAI intermediate phase is expected to form ${ }^{21}$. It is believed that the formation of the
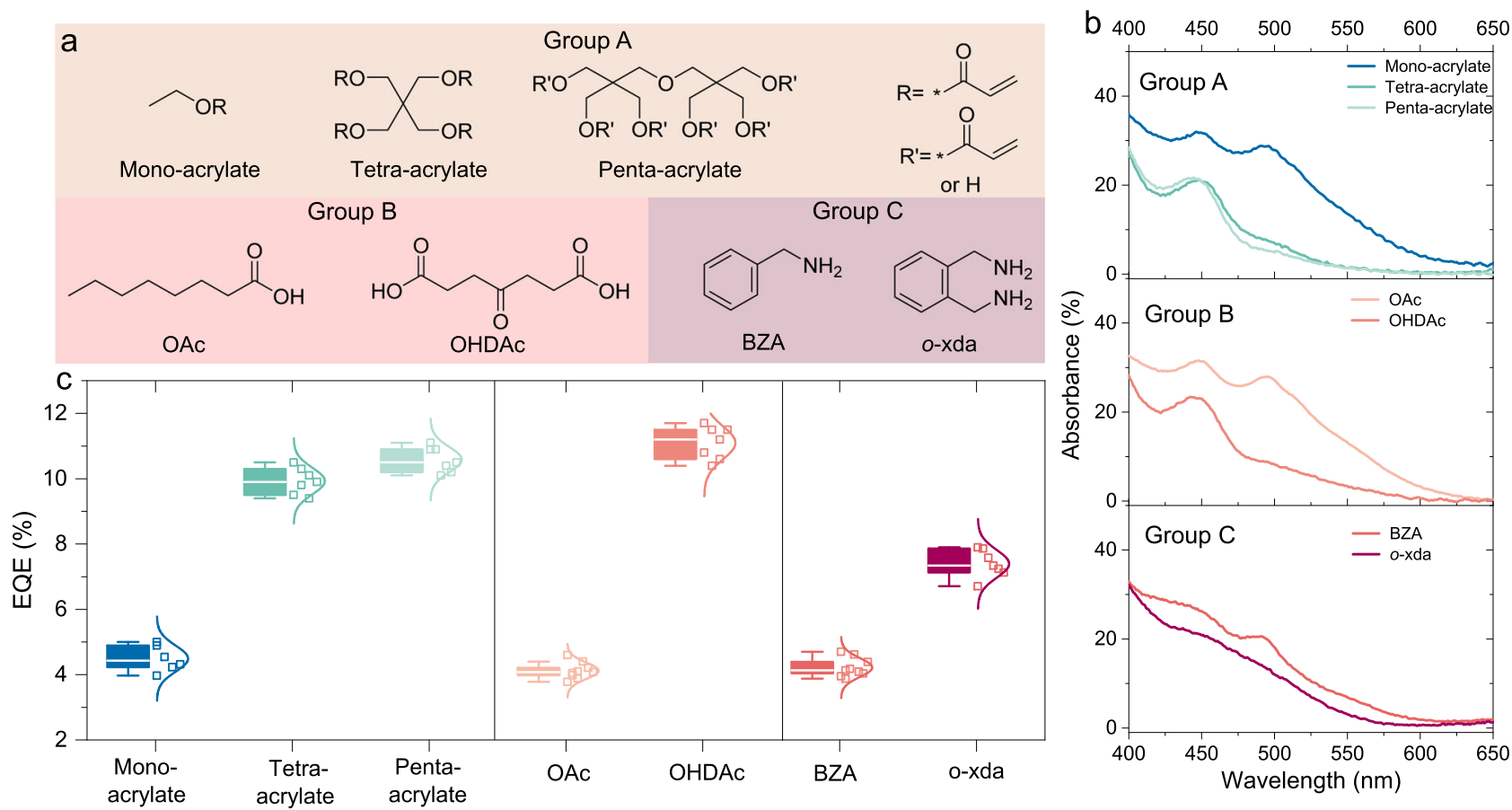

Fig. 4 Dependence of device performance on the coordination number of additives and relevant affinity to lead cations. a Chemical structures of the alternative additives, including acrylates (ethyl acrylate (mono-acrylate), pentaerythritol tetraacrylate (tetraacrylate), and dipentaerythritol penta-acrylate (penta-acrylate), Group A), carboxylic acids (octane acid (OAc) and 4-oxoheptanedioic acid (OHDAc), Group B), and aromatic amines (benzylamine (BZA) and o-xylylenediamine (o-xda), Group C). b Ultraviolet-visible (UV-vis) absorption spectra for the precursor films (Pbla:FAl:additive $=1: 2: x)$ with acrylates $(x=0.80,0.20,0.16$ for mono-, tetra-, and penta-acrylates, respectively, top), carboxylic acids $(x=0.30,0.10$ for OAc and OHDAc, respectively, middle) and aromatic amines ( $x=0.2$ and 0.1 for BZA and o-xda, respectively, bottom). c A summary of average EQE values for the optimized devices based on various additives. 
intermediate phase can retard perovskite crystallization and hence enable high film qualities. In contrast, we observe the appearance of red DMSO-precursor films in our case. The excitonic absorption features in the UV-vis absorption spectrum (Supplementary Fig. 24a) are identical to the films without any additives, indicating the formation of perovskite nanocrystals in the precursor films rather than $\mathrm{DMSO}-\mathrm{PbI}_{2}-\mathrm{FAI}$ intermediate phases. The difference between the DMSO-precursor film in photovoltaics and that in LEDs in our case can be ascribed to the employment of excess FAI and relevant changes in coordination equilibrium in the films. In other words, as a result of the weak DMSO- $\mathrm{PbI}_{2}$ binding affinity, the DMSO- $\mathrm{PbI}_{2}-\mathrm{FAI}$ intermediate phase is not stable in a haliderich environment that is typically used in PeLEDs. Consistently, we observe no positive effect on the device performance of PeLEDs with the DMSO addition (Supplementary Fig. 24).

We proceed to investigate other Lewis base additives with different donating functional moieties and coordination numbers, including acrylates in Group A, carboxylic acids in Group B and aromatic amines in Group C (Fig. 4a). To estimate the dependence of lead-additive affinity on the coordination number of the additives in each group, we measure the UV-vis absorption spectra of the precursor films and keep the total amounts of functional moieties constant in each group by modifying the ratio of additives. With increasing coordination numbers, the absorption signatures from high-order iodoplumbate complex and/or crystalline perovskites gradually decrease (Fig. 4b), indicating enhanced lead-additive binding affinity. Due to the weak interaction between acrylates and lead cations, only those with high coordination numbers (e.g., tetra- and penta-acrylates) can influence the constituents of iodoplumbate complexes within the precursor films. We show the representative device characteristics in Supplementary Fig. 25. The averaged peak EQE values and all the detailed performance parameters are summarized in Fig. 4c and Supplementary Table 2, respectively. As expected, the device performance improves with increasing coordination numbers in each group. In addition, the dependence of device performance on the coordination numbers of additives is also visible in aromatic acids (Supplementary Fig. 26). All these results further confirm that increasing the lead-additive affinity by leveraging the chelate effect is a universal and effective strategy for preparing highly emissive perovskite thin films.

Having concluded the effectiveness and general applicability of chelation enhanced lead-additive affinity on improving film quality and device performance, the remaining question is if there is an upper limit to the affinity strength before film quality no longer benefits. We thus start with the bidentate chelating additive, hexamethylenediamine (HMDA), from which we enhance the affinity strength by moving to a multidentate chelating ligand, that is, triethylenetetramine (Trien) (see molecular structures in Supplementary Fig. 27). Trien is wellknown for its superstrong affinity to metal ions and hence has been widely used to construct environmentally stable metal complexes ${ }^{39}$. Adding Trien into the perovskite precursor solutions leads to the immediate formation of white precipitation (Supplementary Fig. 28), indicating that the lead-Trien binding is too strong to be replaced by iodides. This result also indicates that an additive with too strong affinity may bring about impurities into the films. In contrast, HMDA addition has no negative impact on perovskite formation, and its binding affinity to lead cations is strong enough to suppress perovskite nucleation at room temperature by leveraging the chelate effect (Supplementary Fig. 29). The optimal HMDA-based devices give an average EQE of $\sim 11 \%$ (Supplementary Fig. 30 ). These results indicate that an optimal range of coordination strength should be targeted, with a superstrong lead-additive affinity negatively impacting the perovskite formation.

\section{Discussion}

Based on our collected findings, we present an overview of the correlation between lead-additive coordination affinity and crystallization dynamics in Fig. 5. In all scenarios, the additives compete with halide anions to coordinate with lead cations, resulting in an enhanced activation energy barrier $\left(\Delta E_{a}\right)$ for perovskite nucleation due to the additional interactions involved in the formation of $\mathrm{PbI}_{6}^{4-}$ octahedra. However, the halide-rich environment typically used for fabricating PeLEDs significantly hinders the coordination of additives and the relevant formation of intermediate phases. This becomes dominant for the case of using additives with weak coordination affinities, such as DMSO and the above-mentioned MFAs, resulting in little difference in the energy barrier for perovskite crystallization compared to those without using any additives. Consequently, perovskite nucleation and crystallization occur rapidly at the initial stage during annealing or even happen at room temperature, leading to a high density of defect sites and severe nonradiative recombination. In

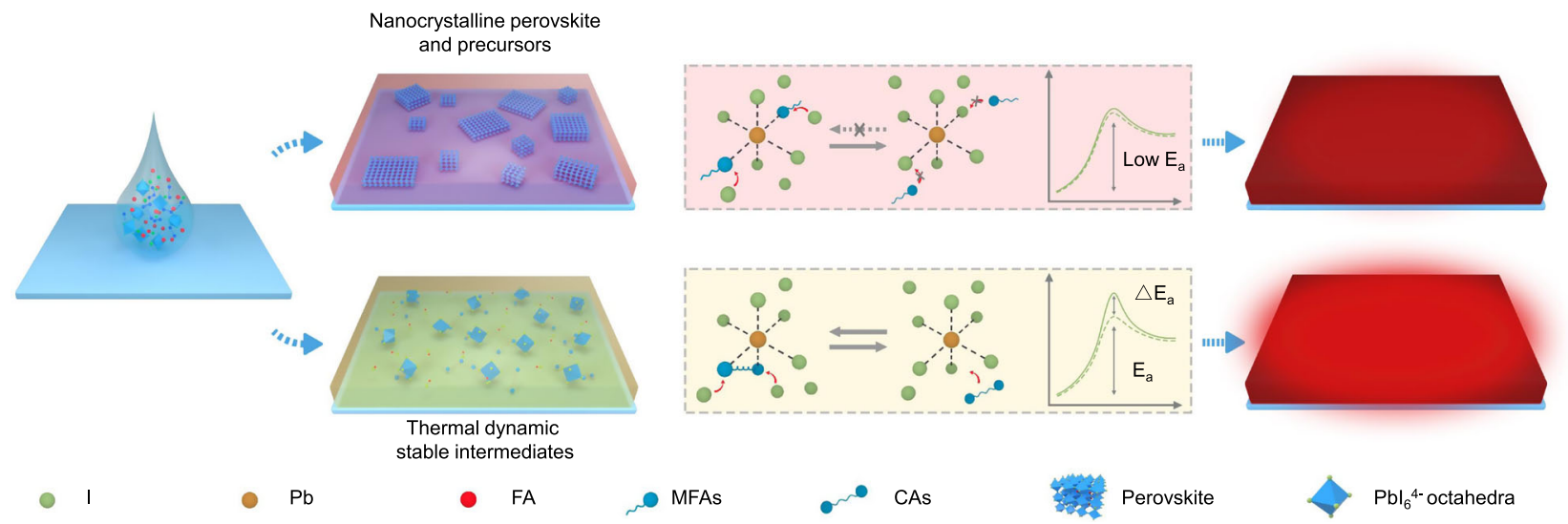

Fig. 5 Schematic illustration of perovskite crystallization process with different lead-additive binding affinity. The additives with weak coordination affinities (e.g., MFAs) cause rapid perovskite nucleation at the initial stage of thermal annealing or even at room temperature. And those with strong affinity (e.g., CAs) bring about thermodynamically stable intermediates, resulting in highly emissive perovskites. The pink and yellow boxes illustrate the competitive ligand exchange between halides and additives with different binding affinity, as well as the respective evolution of thermal energy barrier (solid line) of perovskite crystallization compared to that without using additive (dash line). Here, $E_{a}$ denotes the activation energy for crystallization, and $\Delta E_{a}$ is the enhanced energy caused by additive coordination. 
comparison, the strong lead-CA binding affinity enables effective ligand exchange, which takes over halide coordination. It thus gives rise to an enlarged energy barrier for perovskite nucleation by the formation of more thermodynamically and kinetically stable intermediate phases, slowing down the release of lead cations for perovskite crystallization during the annealing process. We also note that a multidentate additive with superstrong affinity may suppress the formation of perovskite crystals or bring about impurities that deteriorate the device performance. As such, for a specific precursor with a particular halide stoichiometry, tuning the lead-additive coordination affinity will be very useful for achieving optimal crystal quality. From a material design point of view, besides tuning coordination number of chelating molecular discussed here, other factors influencing the stability of metal complexes such as hard-soft nature of functional groups, macrocyclic effect, resonance effect, steric hindrance are all potential protocols for tuning the affinity and relevant crystallization dynamics of perovskites.

In summary, we have elucidated how molecular additives improve electroluminescence of perovskite emitters and developed a complete crystallization model which goes beyond defect passivation, by establishing the correlation between lead-additive coordination affinity and perovskite crystallization dynamics. We conclude that the additives with a strong binding affinity that leads to kinetically and thermodynamically stable intermediate phases are more effective in inhibiting perovskite nucleation and slowing down crystallization dynamics, which enables high crystal quality and thus efficient electroluminescence. This also indicates a different requirement in lead-additive binding affinity between perovskite emitters and photovoltaic absorbers due to the discrepancies in precursor stoichiometry. Our results rationalize the general and puzzling observation that chelating additives are much more widely used in state-of-the-art PeLEDs, and provide a universal guideline for tuning the crystallization dynamics for high-performance PeLEDs by leveraging the chelate effect.

\section{Methods \\ Materials. Lead iodide $\left(\mathrm{PbI}_{2}\right.$, anhydrous, $\left.99.999 \%\right)$ was purchased from Alfa Aesar. Formamidinium iodide (FAI) was purchased from GreatCell Solar. Poly $(9,9-$ dioctyl-fluorene-co-N-(4-butylphenyl)diphenylamine) (TFB) was purchased from American Dye Source. 2-(2-methoxyethoxy) ethanamine ( $\left.\mathrm{m}-\mathrm{PEG}_{2}-\mathrm{NH}_{2}\right), 2,5,8,11$ - tetraoxatridecan-13-amine $\left(\mathrm{m}-\mathrm{PEG}_{4}-\mathrm{NH}_{2}\right)$ and 3,6,9,12-tetraoxatetradecane-1,14- diamine $\left(\mathrm{NH}_{2}-\mathrm{PEG}_{4}-\mathrm{NH}_{2}\right)$ were obtained from Biochempeg. Ethyl acrylate (mono-acrylate), pentaerythritol tetraacrylate (tetraacrylate), dipentaerythritol penta-acrylate (penta-acrylate), octane acid (OAc), 4-oxoheptanedioic acid (OHDAc), hexamethylenediaminedie (HMDA), triethylenetetramine (Trien), and all the other materials were purchased from Sigma-Aldrich. $\mathrm{ZnO}$ nanoparticles were synthesized as reported in the literature ${ }^{40}$.}

Preparation of perovskite solution. All the additives were introduced into perovskite precursors with a mole ratio of $\mathrm{PbI}_{2}$ :formamidinium iodide (FAI):additives $=1: 2: x(x=0-0.8)$ in DMF to prepare the perovskite precursor solutions. The optimized feed ratio of additives for each type of device has been summarized in Supplementary Table 1.

Device fabrication. Patterned ITO glass substrates were cleaned with deionized water and ethanol step by step, followed by UV-ozone treatment for $20 \mathrm{~min}$ before use. $\mathrm{ZnO}$ nanoparticles were spin-coated on the cleaned ITO substrates with 3000 r.p.m. for $30 \mathrm{~s}$ in ambient. Then, the $\mathrm{ZnO}$-coated substrates were moved into an $\mathrm{N}_{2}$-filled glove box to deposit PEIE ( $1.1 \mathrm{mg} \mathrm{mL}-1$ in isopropanol) at 3000 r.p.m. for $30 \mathrm{~s}$, followed by annealing at $100^{\circ} \mathrm{C}$ for $10 \mathrm{~min}$. After cooling down, perovskite precursors were spin-coated at 3000 r.p.m. for $30 \mathrm{~s}$ and then annealed on a preheated hotplate at $100^{\circ} \mathrm{C}$ for $10 \mathrm{~min}$. TFB $\left(12 \mathrm{mg} \mathrm{mL}^{-1}\right.$ in chlorobenzene) was spin-coated on top of the perovskite films at 3000 r.p.m. for 30 s. After that, $7 \mathrm{~nm}$ $\mathrm{MoO}_{3}$ and $80 \mathrm{~nm} \mathrm{Au}$ were deposited as the electrode in a thermal evaporator, the deposition rates are $\sim 0.2$ and $\sim 0.5 \AA \mathrm{s}^{-1}$, respectively. For the electron-only devices, phenyl-C61-butyric acid methyl ester (PCBM, $30 \mathrm{mg} \mathrm{mL}^{-1}$ in chlorobenzene) was spin-coated on the top of the perovskite films at 3000 r.m.p for $30 \mathrm{~s}$, followed by annealing at $70^{\circ} \mathrm{C}$ for $10 \mathrm{~min}$, followed by deposition of $100 \mathrm{~nm} \mathrm{Al}$. The active area of our PeLEDs is $7.25 \mathrm{~mm}^{2}$.
Device characterization. The PeLEDs were measured at room temperature in an $\mathrm{N}_{2}$-filled glove box. A Keithley 2400 source meter incorporated with a QE Pro spectrometer (Ocean Optics) was used to measure the electrical output of PeLEDs. The applied voltage started from $0 \mathrm{~V}$ and increased with a step of $0.05 \mathrm{~V}$, lasting for $300 \mathrm{~ms}$ at each voltage step for stabilization and measurements. The operational stability of the PeLEDs was measured under a constant current density of $20 \mathrm{~mA}$ $\mathrm{cm}^{-2}$ in the glove box.

Grazing incident wide-angle X-ray scattering (GIWAXS). GIWAXS data were obtained from NCD-SWEET beamline, ALBA Synchrotron, Spain. The energy of the X-ray beam was set to $12.95 \mathrm{keV}$ using a Si $\left(\begin{array}{llll}1 & 1 & 1\end{array}\right)$ channel-cut monochromator and further collimated with an array of Be lenses. The incidence angle was $1^{\circ}$ and the diffraction patterns were collected using a Rayonix ${ }^{\circ}$ LX255-HS area detector, which consists of a pixel array of $5760 \times 1920(\mathrm{~V} \times \mathrm{H})$ with a pixel size of $88.54 \times$ $88.54 \mu \mathrm{m}^{2}$ for the pixel binning employed of $2 \times 2$. The scattering vector $q$ was calibrated using $\mathrm{Cr}_{2} \mathrm{O}_{3}$ as standard, obtained using a sample to detector distance of $220 \mathrm{~mm}$. All the samples were measured using a calibrated Linkam THMS600 heating stage adapted for grazing-incidence experiments under $\mathrm{N}_{2}$ atmosphere. The scan rate was one frame per two seconds.

Transient absorption (TA). The femtosecond transient absorption spectra were collected using a pump-probe configuration. A femtosecond oscillator (Mai Tai, Spectra Physics) is used as a seed laser and injected into a regenerative amplifier (Spitfire XP Pro, Spectra Physics) to generate a pulsed laser $(800 \mathrm{~nm}, 80 \mathrm{fs}$ pulse duration, $1 \mathrm{kHz}$ repetition frequency). The second-harmonic generation $(400 \mathrm{~nm})$ from a BBO crystal was used as the pump. And the white light continuum (WLC) as the probe was produced by focusing the $800 \mathrm{~nm}$ fs pulse on a thin $\mathrm{CaF}_{2}$ plate. Polarization between the pump and probe was set to the magic angle $\left(54.7^{\circ}\right)$. During the experiments, gates on the pump intensity and probe spectra were set to avoid the influence of laser fluctuation on the measurements.

Thin-film characterization. X-ray diffraction (XRD) patterns were conducted by an X-ray diffractometer (Panalytical X'Pert Pro) with $\mathrm{CuK} \alpha$ radiation. The X-ray was generated on a Copper target, and the wavelength of the X-ray was $1.54 \AA$. Morphology images of the perovskite films were measured by an FEI (Quanta 200 FEG) scanning electron microscopy under a voltage of $5 \mathrm{kV}$. UV-vis absorbance spectra were collected from a PerkinElmer Lambda 900. All the precursor films for UV-vis measurements were encapsulated by glass slides and epoxy. Steady-state PL spectra were obtained with a 450-nm excitation laser and an Andor spectrometer (Shamrock sr-303i-B, coupled to a Newton EMCCD Si array detector). The fluence-dependent PLQEs were measured by a typical three-step technique with a combination of a 445-nm continuous-wave laser, spectrometer, and an integrating sphere.

The PL mapping measurements were conducted from a home-built wide-field epifluorescence microscope based on Olympus IX71 with a $\times 40$ objective lens (Olympus LUCPlanFL, NA = 0.6). A 405-nm diode laser $(\mathrm{CW})$ was used for excitation. The power density was approximately $0.25 \mathrm{~W} \mathrm{~cm}^{-2}$. The PL intensity as a function of time was recorded by a charge-coupled device camera (ProEM 512B, Princeton Instruments) with $100 \mathrm{~ms}$ of exposure time.

Fourier transform-infrared (FT-IR) spectra of perovskite films on ITO/ZnO/ PEIE were conducted by a PIKE MIRacle Vertex 70 Spectrometer (Bruker) using a DLaTGS detector at room temperature. The spectrometer is equipped with a PIKE MIRacle $^{\mathrm{TM}}$ attenuated total reflectance (ATR) accessory as the sampling accessory. The measuring system was continuously purged with $\mathrm{N}_{2}$ before and during the measurements. The spectra were acquired at $2 \mathrm{~cm}^{-1}$ resolution with 30 scans over a wavenumber range between 4000 and $600 \mathrm{~cm}^{-1}$.

Transmittance electron microscopy (TEM) of precursor films was performed by a TECNAI G2 F20 transmission electron microscope with an accelerating voltage of $200 \mathrm{kV}$ and a Gatan SC200 CCD camera. Ultraviolet photoelectron spectroscopy (UPS) of perovskite films were recorded by a multifunctional photoelectron spectrometer (PHI 5000 Versaprobe II) with a He I ultraviolet radiation source $(21.2 \mathrm{eV})$.

Nuclear magnetic resonance (NMR). High-resolution ${ }^{1} \mathrm{H}$ NMR data were recorded on a Bruker $600 \mathrm{MHz}$ Avance III spectrometer at Larmor frequencies $v$ $\left({ }^{1} \mathrm{H}\right)=600.274 \mathrm{MHz} .{ }^{1} \mathrm{H}$ NMR measurements single pulse experiments with $90^{\circ}$ pulse of $18 \mu \mathrm{s}$, relaxation delay $10 \mathrm{~s}, 64$ scans were applied. Dried $\mathrm{D}^{7}$-DMF (99.5\%) was used as the solvent and was also used to calibrate isotropic chemical shifts. The temperature was kept constant at $298 \pm 0.2 \mathrm{~K}$ with a BVT 3000 temperature unit during measurements. All samples were dissolved in dried DMF at room temperature in an inert (Ar) atmosphere. The concentration for $\mathrm{Pb}^{2+}$ and $\mathrm{FAI}$ is 0.12 and $0.24 \mathrm{M}$, respectively.

UV-vis absorbance spectra for solution and Benesi-Hildebrand (BH) analyses. UV-vis absorbance spectra for solutions were collected by a PerkinElmer Lambda 35 at room temperature. Anhydrous DMF was used as the solvent. The concentration of $\mathrm{Pb}^{2+}$ for all the solutions is fixed at $0.25 \mathrm{mM}$. The mole ratio of $\mathrm{PbI}_{2}: \mathrm{m}-\mathrm{PEG}_{2}-\mathrm{NH}_{2}$ and $\mathrm{PbI}_{2}: \mathrm{NH}_{2}-\mathrm{PEG}_{4}-\mathrm{NH}_{2}$ parent solution is $1: 10$ and $1: 5$, respectively. The formation equilibrium constant $\left(\mathrm{K}_{\mathrm{f}}\right)$ of $\mathrm{PbI}_{3}{ }^{-}$was deduced by the 
Benesi-Hildebrand equation stated below:

$$
\frac{1}{A-A_{0}}=\frac{1}{K_{f}\left(A_{\max }-A_{0}\right)[\mathrm{FAI}]}+\frac{1}{A_{\max }-A_{0}}
$$

Here, $A_{0}$ is $\mathrm{PbI}_{3}{ }^{-}$absorbance of the parent solution without FAI addition; $A$ is the collected absorbance with FAI; $A_{\max }$ is the saturated absorbance with FAI addition; $K_{\mathrm{f}}$ is deduced from the slope of Benesi-Hildebrand plot.

\section{Density-functional theory (DFT) calculations. We performed the density-} functional theory (DFT) calculations by using the projector-augmented wave (PAW) method as implemented in Vienna Ab initio Simulation Package (VASP) ${ }^{41,42}$. The generalized gradient approximation (GGA) with Perdew-Burke-Ernzerhof (PBE) method was used to optimize the crystal structures of cubic-phase $\mathrm{FAPbI}_{3}$ bulk, slabs, and interfaces. The wave functions were expanded on a plane-wave basis set up to a kinetic energy cutoff of $450 \mathrm{eV}$. The van der Waals functional (vdw) was also included in the structural optimizations and electronic calculations using the zero-damping DFT-D3 method of Grimme. Monkhorst-Pack-type K-meshes of $6 \times 6 \times 6$ for the bulk $\mathrm{FAPbI}_{3}, 4 \times 4 \times 1$ for the $\mathrm{FAPbI}_{3}$ (110) slabs with different terminations, and $2 \times 2 \times 1$ for the super-slabs and interfaces with $\mathrm{m}-\mathrm{PEG}_{2}-\mathrm{NH}_{2}$ or $\mathrm{m}-\mathrm{PEG}_{4}-\mathrm{NH}_{2}$ modification. All the structures were optimized until the forces on every single atom were less than $0.01 \mathrm{eV} / \AA$. The binding energies were defined as $E_{\mathrm{B}}=E_{\text {interface }}-E_{\mathrm{FAPbI} 3}-E_{\text {ligand }}$, where $E_{\text {interface }}$ is the total energy of the interface after $\mathrm{m}-\mathrm{PEG}_{2}-\mathrm{NH}_{2}$ or $\mathrm{m}-\mathrm{PEG}_{4}-\mathrm{NH}_{2}$ passivation, and $E_{\mathrm{FAPb} 3}$ and $E_{\text {ligand }}$ represent the total energies of the individual parts. The molecular graphics viewer VESTA was used to plot the crystal structures.

The geometrical and electronic properties of additives were calculated by B3LYP (Becke Three Parameters Hybrid Functional with Lee-Yang-Perdew correlation functions) functional and 6-31G* basis set with Gaussian 09 program package. The electrostatic potential surfaces were visualized and drawn using Multiwfn and VMD software.

\section{Data availability}

The data that support the plots within this paper and other findings of this study are available from the corresponding author upon reasonable request.

Received: 22 January 2021; Accepted: 20 July 2021;

Published online: 10 August 2021

\section{References}

1. Liu, X.-K. et al. Metal halide perovskites for light-emitting diodes. Nat. Mater. 20, 10-21 (2021)

2. Tan, Z.-K. et al. Bright light-emitting diodes based on organometal halide perovskite. Nat. Nanotechnol. 9, 687-692 (2014).

3. Protesescu, L. et al. Nanocrystals of cesium lead halide perovskites $(\mathrm{CsPbX}$, $\mathrm{X}=\mathrm{Cl}, \mathrm{Br}$, and I): novel optoelectronic materials showing bright emission with wide color gamut. Nano Lett. 15, 3692-3696 (2015).

4. Xiao, Z. et al. Efficient perovskite light-emitting diodes featuring nanometresized crystallites. Nat. Photon. 11, 108-115 (2017).

5. Lin, K. et al. Perovskite light-emitting diodes with external quantum efficiency exceeding 20 percent. Nature 562, 245-248 (2018).

6. Cao, Y. et al. Perovskite light-emitting diodes based on spontaneously formed submicrometre-scale structures. Nature 562, 249-253 (2018).

7. Xu, W. et al. Rational molecular passivation for high-performance perovskite light-emitting diodes. Nat. Photon. 13, 418-424 (2019).

8. Ban, M. et al. Solution-processed perovskite light-emitting diodes with efficiency exceeding $15 \%$ through additive-controlled nanostructure tailoring. Nat. Commun. 9, 3892 (2018).

9. Braly, I. L. et al. Hybrid perovskite films approaching the radiative limit with over 90\% photoluminescence quantum efficiency. Nat. Photon. 12, 355-361 (2018).

10. Abdi-Jalebi, M. et al. Maximizing and stabilizing luminescence from halide perovskites with potassium passivation. Nature 555, 497-501 (2018).

11. Noel, N. K. et al. Enhanced photoluminescence and solar cell performance via Lewis base passivation of organic-inorganic lead halide perovskites. ACS Nano 8, 9815-9821 (2014).

12. Jiang, Y. et al. Spectra stable blue perovskite light-emitting diodes. Nat. Commun. 10, 1868 (2019).

13. Yang, X. et al. Efficient green light-emitting diodes based on quasi-twodimensional composition and phase engineered perovskite with surface passivation. Nat. Commun. 9, 570 (2018).

14. Karlsson, M. et al. Mixed halide perovskites for spectrally stable and highefficiency blue light-emitting diodes. Nat. Commun. 12, 361 (2021)

15. Chen, B. et al. Imperfections and their passivation in halide perovskite solar cells. Chem. Soc. Rev. 48, 3842-3867 (2019).

16. Nenon, D. P. et al. Design principles for trap-free $\mathrm{CsPbX}$ nanocrystals enumerating and eliminating surface halide vacancies with softer Lewis bases. J. Am. Chem. Soc. 140, 17760-17772 (2018).
17. Wang, F. et al. Phenylalkylamine passivation of organolead halide perovskites enabling high-efficiency and air-stable photovoltaic cells. Adv. Mater. 28, 9986-9992 (2016)

18. Liu, S. et al. A review on additives for halide perovskite solar cells. Adv. Energy Mater. 10, 1902492 (2020).

19. Zhang, F. et al. Additive engineering for efficient and stable perovskite solar cells. Adv. Energy Mater. 10, 1902579 (2020).

20. Wang, H. et al. Perovskite-molecule composite thin films for efficient and stable light-emitting diodes. Nat. Commun. 11, 891 (2020).

21. Jeon, N. J. et al. Solvent engineering for high-performance inorganic-organic hybrid perovskite solar cells. Nat. Mater. 13, 897-903 (2014).

22. Deng, Y. et al. Tailoring solvent coordination for high-speed, roomtemperature blading of perovskite photovoltaic films. Sci. Adv. 5, eaax7537 (2019).

23. Lee, J.-W. et al. Tuning molecular interactions for highly reproducible and efficient formamidinium perovskite solar cells via adduct approach. J. Am. Chem. Soc. 140, 6317-6324 (2018).

24. Yuan, M. et al. Perovskite energy funnels for efficient light-emitting diodes. Nat. Nanotechnol. 11, 872-877 (2016).

25. Liu, Y. et al. Efficient blue light-emitting diodes based on quantum-confined bromide perovskite nanostructures. Nat. Photon. 13, 760-764 (2019).

26. Li, J. et al. 50-Fold EQE improvement up to $6.27 \%$ of solution-processed allinorganic perovskite $\mathrm{CsPbBr}_{3}$ QLEDs via surface ligand density control. $A d v$. Mater. 29, 1603885 (2017).

27. Cho, H. et al. Overcoming the electroluminescence efficiency limitations of perovskite light-emitting diodes. Science 350, 1222-1225 (2015).

28. Yuan, Z. et al. Unveiling the synergistic effect of precursor stoichiometry and interfacial reactions for perovskite light-emitting diodes. Nat. Commun. 10, 2818 (2019)

29. Swarnkar, A. et al. Colloidal $\mathrm{CsPbBr}$ perovskite nanocrystals: luminescence beyond traditional quantum dots. Angew. Chem. Int. Ed. 127, 15644-15648 (2015).

30. Gerhard, M. et al. Microscopic insight into non-radiative decay in perovskite semiconductors from temperature-dependent luminescence blinking. Nat. Commun. 10, 1698 (2019).

31. Xing, G. et al. Long-range balanced electron- and hole-transport lengths in organic-inorganic $\mathrm{CH}_{3} \mathrm{NH}_{3} \mathrm{PbI}_{3}$. Science 342, 344 (2013).

32. Christians, J. A. et al. Transformation of the excited state and photovoltaic efficiency of $\mathrm{CH}_{3} \mathrm{NH}_{3} \mathrm{PbI}_{3}$ perovskite upon controlled exposure to humidified air. J. Am. Chem. Soc. 137, 1530-1538 (2015).

33. Zheng, K. et al. High excitation intensity opens a new trapping channel in organic-inorganic hybrid perovskite nanoparticles. ACS Energy Lett. 1, 1154-1161 (2016).

34. Ahn, N. et al. Highly reproducible perovskite solar cells with average efficiency of $18.3 \%$ and best efficiency of $19.7 \%$ fabricated via Lewis base adduct of lead (II) iodide. J. Am. Chem. Soc. 137, 8696-8699 (2015).

35. Li, J. et al. Phase transition control for high-performance blade-coated perovskite solar cells. Joule 2, 1313-1330 (2018).

36. Hamill, J. C. et al. Influence of solvent coordination on hybrid organic-inorganic perovskite formation. ACS Energy Lett. 3, 92-97 (2018).

37. Kuntz, I. Jr et al. Molecular interactions and the Benesi-Hildebrand equation. J. Am. Chem. Soc. 90, 4778-4781 (1968).

38. $\mathrm{Hu}, \mathrm{Q}$. et al. In situ dynamic observations of perovskite crystallisation and microstructure evolution intermediated from $\left[\mathrm{PbI}_{6}\right]^{4-}$ cage nanoparticles. Nat. Commun. 8, 15688 (2017).

39. Pecsok, R. L. et al. Complexes of chromium (II) with diethylenetriamine and triethylenetetramine. Inorg. Chem. 4, 447-451 (1965).

40. Bai, S. et al. High-performance planar heterojunction perovskite solar cells: preserving long charge carrier diffusion lengths and interfacial engineering. Nano Res. 7, 1749-1758 (2014).

41. Kresse, G. et al. Efficient iterative schemes for ab initio total-energy calculations using a plane-wave basis set. Phys. Rev. B 54, 11169-11186 (1996).

42. Kresse, G. et al. From ultrasoft pseudopotentials to the projector augmentedwave method. Phys. Rev. B 59, 1758-1775 (1999).

\section{Acknowledgements}

The authors thank Prof. Annamaria Petrozza, Dr. Tiankai Zhang for valuable discussions, and Kaichuan Wen and Prof. Jianpu Wang for help with the PLQE measurements We acknowledge the support from the ERC Starting Grant (No. 717026), the Swedish Energy Agency Energimyndigheten (Nos. 48758-1 and 44651-1), Swedish Research Council VR, NanoLund, and the Swedish Government Strategic Research Area in Materials Science on Functional Materials at Linköping University (Faculty Grant SFOMat-LiU No. 2009-00971). The authors would like to thank the NCD-SWEET beamline at ALBA Synchrotron from Spain for providing the beamtime. J.Y, O.F.M., and O.M.B. acknowledge the Supercomputing Laboratory at KAUST for their computational and storage resources. Y.Z., L.C., and B.S. thanks the National Natural Science Foundation of China (91833303, 61974098, 62005126), the National Key Research and Development Program (2016YFA0201900), Jiangsu High Educational Natural Science Foundation 
(18KJA430012), the 111 Program and Collaborative Innovation Center of Suzhou Nano Science and Technology, and Collaborative Innovation Center of Suzhou Nano Science \& Technology. J.A.S. acknowledges financial support from the Research Foundation Flanders (FWO: grant No.'s 12Y7218N and 12Y7221N). M.B.J.R. acknowledges financial support from the Research Foundation - Flanders (FWO, G098319N) and the KU Leuven Research Fund (C14/19/079). Y.Z. (No. 201806920071), P.T. (No. 201906830040, J.L. (No. 201608530162), W.L. (No. 201806460021) also thank the financial support from China Scholarship Council. F.G. is a Wallenberg Academy Fellow.

\section{Author contributions}

F.G. and W.X. conceived the idea and supervised the project; Y.Z., P.T., and W.X. performed the experiments and analyzed the data; G.Z., J.A.S., E.S., and M.B.J.R. performed in situ GIWAXS measurements and analyzed the data; W.L. performed transient absorption under the supervision of K.Z. and T.P.; J.Y. performed DFT calculation for understanding the passivation effectiveness under supervision of O.F.M. and O.M.B., L.K., S.A., and J.B. performed NMR tests and analyzed the data. X.L. performed UV-vis absorption tests and contributed to $\mathrm{BH}$ analysis. J.L. performed PL mapping tests under the supervision of I.S.; C.K. optimized the synthesis of ZnO. L.C. performed DFT calculation under the supervision of B.S. B.S. supervised Y.Z., Y.Y. supervised P.T., B.S and S.B. contributed to the interpretation of results; W.X., Y.Z., and F.G. wrote the manuscript; B.S. and J.S. provided revisions to the manuscript. All authors discussed the results and commented on the manuscript.

\section{Funding}

Open access funding provided by Linköping University.

\section{Competing interests}

The authors declare no competing interests.

\section{Additional information}

Supplementary information The online version contains supplementary material available at https://doi.org/10.1038/s41467-021-25092-7.

Correspondence and requests for materials should be addressed to W.X., B.S. or F.G.

Peer review information Nature Communications thanks Biwu Ma and the other, anonymous, reviewer(s) for their contribution to the peer review of this work.

Reprints and permission information is available at http://www.nature.com/reprints

Publisher's note Springer Nature remains neutral with regard to jurisdictional claims in published maps and institutional affiliations.

(c) Open Access This article is licensed under a Creative Commons Attribution 4.0 International License, which permits use, sharing, adaptation, distribution and reproduction in any medium or format, as long as you give appropriate credit to the original author(s) and the source, provide a link to the Creative Commons license, and indicate if changes were made. The images or other third party material in this article are included in the article's Creative Commons license, unless indicated otherwise in a credit line to the material. If material is not included in the article's Creative Commons license and your intended use is not permitted by statutory regulation or exceeds the permitted use, you will need to obtain permission directly from the copyright holder. To view a copy of this license, visit http://creativecommons.org/ licenses/by/4.0/.

(C) The Author(s) 2021 